both in girls and boys. Also, it was the leading FGID in the infant/toddler (41/57) and the children group (66/124). Retentive encopresis was present in about $1 / 5$ of children $(29 / 132$ or $21.9 \%$ ) Accompanied encopresis was mainly observed in the age group of $4-10$ years $\left(22 / 66\right.$ or $33.3 \%, \chi^{2}=9.9431$, $\mathrm{p}<0.05)$. Also, encopresis tends to be more frequent among male patients with functional constipation (male, $27 \%$ vs female, 15.5\%). In patients who visited PG for the first time, substantial discordance in the referral and the final diagnosis was observed. About $1 / 3$ of patients with diagnosed constipation was referred with another diagnosis (29/92 or $31.5 \%$ ). However, most of patients with constipation as referral diagnosis had the same disorder as final diagnosis (49/53 or 92.5\%).

Discussion Frequency of functional constipation in paediatric patients in tertiary hospital setting, as well as sex and age distribution, is comparable to reported incidences in the population. The discordance of referral and final diagnosis shows that constipation is not adequately recognized in primary care, but when recognized, it is mostly diagnosed correctly.

Conclusion Functional constipation is not recognized enough in primary care and more efforts are needed to assure a positive diagnosis of this disorder prior to the referral to tertiary care.

\section{P606 TRANSIENT CHOLESTASIS IN NEONATAL INTENSIVE CARE UNIT (ABOUT 46 CASES)}

Chiraz Regaieg*, Amel Ben Hmed, Mouna Loukil, Manel Charfi, Amira Bouraoui, Nedia Hmida, Ridha Regaieg, Afef Ben Thabet, Abdellatif Gargouri. Department of Neonatology, Hedi Chaker Hospital, Sfax, Tunisia

\subsection{6/archdischild-2019-epa.938}

Background Transient cholestasis is common in neonatal intensive care unit. It accounts for 5 to $10 \%$ of neonatal cholestasis.

Objectives To describe the characteristics of neonatal cholestasis and to identify the etiology and associated factors.

Methods It's a retrospective study registering 46 cases of transient cholestasis in the neonatology department of Sfax from 2010 to 2018.

Results A great prematurity was noted in half of the cases as well as a hypotrophy. Perinatal asphyxia was noted in $41 \%$ of cases. Nosocomial infection was present in $50 \%$ of cases and severe hemodynamic disorders in $9 \%$ of cases. Four full term newborns had received intensive phototherapy. The average of parenteral nutrition duration was 18 days (1-64 days). The mean age at the time of diagnosis of the cholestasis was 29 days (3 to 69 days). Abdominal ultrasound showed vesicular lithiasis in two cases, portal vein thrombosis in two cases, and anaerobic abscess in one case. Etiological explorations were normal in all other cases. Follow up showed a disappearance of cholestasis and the restoration of liver function after 2 to 3 months. Finally, this transient cholestasis was related to an inspissated bile syndrome in the 4 full term newborns and a portal vein thrombosis in 2 cases. In the other cases, it was associated with prematurity with multifactorial mechanisms including hepatic immaturity, ischemic lesions secondary to perinatal asphyxia and hemodynamic disorders, parenteral nutrition and infections.

Conclusions The results of our analysis suggest that most of these cases of transient neonatal cholestasis may result from the association of several factors including immaturity of bile secretion because of prematurity and perinatal insults leading to hepatic hypoxia or ischemia. Finally, the diagnosis of transient neonatal cholestasis should be considered only with the most extreme caution and after a careful and prolonged follow-up. It is mandatory to rule out other causes of neonatal cholestasis by conducting the appropriate investigations.

\section{P607 PERFORATED ULCER IN A 3 MONTHS AGED INFANT}

Faiza Safi, Lamia Gargouri, Manel Hsairi, Chiraz Regaieg*, Sahar Trichilli, Abdelmajid Mahfoudh. Hedi Chaker Hospital, Sfax, Tunisia

\subsection{6/archdischild-2019-epa.939}

Introduction The digestive manifestations of stress are polymorphic. Gastric or duodenal ulcer is the most characteristic expression. We report the rare case of an infant with severe dehydration complicated by digestive perforation.

Case report A 3 months aged infant was admitted for diarrhea and vomiting evolving since 4 days. She was apathic with severe dehydration. In the biology, she has a hypochromic microcytic anemia, a hyponatremia, an onset of functional renal insufficiency and an infectious syndrome. A triple antibiotic therapy with intravenous rehydration was administrated.

The initial evolution was marked by the improvement of its state of hydration and correction of hydro-electrolytic disorders with secondary appearance of abdominal bloating. Imagery showed pneumoperitoneum at radio and mixed air and fluid intraperitoneal effusion which deals with the diagnosis of digestive perforation.

The baby was operated urgently The peroperative diagnosis was a gastric perforation secondary to a stress ulcer most likely. The postoperative course was simple. The reintroduction of breastfeeding was gradual with good tolerance.

Conclusion The perforation of the stress ulcer is a very rare complication in the infant that must be evoked in front of a pneumoperitoneum. The treatment is always surgical. The prognosis is primarily related to the speed of diagnosis and management. The best treatment is preventive.

\section{LB P608 AN ATYPICAL PRESENTATION OF CROHN'S DISEASE WITH ACUTE JOINT SWELLING}

Qasim Mahmood, Uzair Shabbir*, Irina Chistol, Khalid Khan. University Hospital Kerry, Tralee, Ireland

\subsection{6/archdischild-2019-epa.940}

We present an unusual case of a 9-year-old boy, who attended our emergency department with acute left knee swelling, abdominal pain and fever for the past two days. He was well known to our department with a history of constipation. After Orthopaedic, Surgical and Paediatric review a provisional diagnosis of septic arthritis was made. Later, a diagnosis of Crohn's disease was confirmed and treated accordingly, with the improvement of his symptoms.

This case highlights the importance of exploring vague symptoms of inflammatory bowel disease (IBD) at presentation. Knowledge of extra intestinal manifestations (EIM) is essential. Providers should be able to recognize the unusual manifestations of IBD and initiate an appropriate and timely evaluation. 